\title{
Stability Conditions and the Single Mode Approximation in FQHE
}

\author{
Alejandro Cabo* \\ International Centre for Theoretical Physics, \\ P.O.Box. 586,34100, Trieste, Italy \\ Aurora Pérez-Martinez \\ Grupo de Física Teórica, Instituto de Cibernética, Matemática y Física, \\ Calle E No.309 Esq. a 15, Vedado, La Habana 4, Cuba
}

October 15, 2018

\begin{abstract}
A thermodynamic stability criterion for the spontaneous breaking of translation invariance in many particle systems is derived. It simply requires the positive character of the wavevector dependent dielectric function, generalising the same condition for macroscopic dielectric constants. Its application to the Single Mode Approximation (SMA) for the description of collective modes of the $\nu=1 / \mathrm{m}$ Laughlin states is considered. The results indicate that the stability condition is violated by the SMA for all the relevant states $m=3,5,7,9$ in a wavevector neighborhood of the magneto-roton minima. These conclusions are in qualitative agreement with similar results obtained from the solution of the Bethe-Salpeter equation at $\nu=1 / 3$ for both composite fermions and phenomenologically described electrons in the Laughlin state.
\end{abstract}

*Permanent Address: Grupo de Física Teórica, Instituto de Cibernética, Matemática y Física, Calle E, No. 309, Esq. a 15, Vedado, La Habana 4, Cuba 
The stability properties of quantum many body ground states are of great interest in condensed matter theory. Important questions such as the occurrence of spontaneous breaking of translation, and other, symmetries are closely linked with issues of stabilty. However, the complicated nature of the exact Green function equations for these problems usually makes extracting information about stability difficult. A particular important example includes the determination of the conditions for the formation of the Wigner crystal at low electron gas density, both with and without a magnetic field [1], [2].

In the present work, we discuss the derivation of a requirement to discuss the stability of a translationally invariant many particle system with respect to the spontaneous breaking of this symmetry. In short, this result is a generalisation of the usual positive value condition on the dielectric constant [3] to the case of a general wavevector dependent dielectric function in a translationally invariant many body state.

We expect this constraint may also be helpful in rigorously predicting the breaking of translation invariance which could be necessary to explain short range order in liquids that have fully invariant hamiltonians [ [⿴囗十 $]$.

We apply the criterion to check the so called Single Mode Approximation (SMA) calculations of the collective mode excitations of the Laughlin ground states for $1 / \mathrm{m}$ values of the filling factor [5].

The results indicate that the stability rule is not satisfied in a neighborhood of the magneto-roton minima for the states corresponding to $m=3,5,7,9$. The instability appears to be stronger at lower filling factors as is natural to expect.

These conclusions are in qualitative agreement with our previous results [6], [7]. In [6], the collective mode dispersion for composite fermions at $\nu=1 / 3$ was calculated numerically using the methods in $[\mathbb{8}$. This work predicted an instability region in qualitative agreement with the one obtained here. In place of a magneto-roton minimum. the squared frequency crossed the zero frequency axis as the momentum grew 
from zero, and later became positive at higher momenta.

In [7], we also considered the collective mode dispersion by using a phenomenological ansatz for the exact one electron Green function. It was also assumed that because the mean spatial separation between electrons is of the order of the expected size of the collective mode, screening effects in the interaction kernel of the Bethe-Salpeter equation were small. A region similar to the one found in [6] and in the present work was obtained.

It should be emphasized that the presence of an instability for these fractions does not necessarily mean that the Laughlin or composite fermion descriptions of these systems should be limited. These instabilities could be necessary for completing the picture given by the composite fermion description in which complementary flux quanta are associated with fermions in spatially wide approximate one particle Wannier states. This point of view was advanced in previous works [9], [10], [11] by one of the authors (A.C). However, its validity needs to be supported by the evaluation of ground state energies competitive with the ones corresponding to the Laughlin or composite fermion states.

Moreover, other developments in the literature seem to support the spontaneous breaking of continous translation invariance in FQHE ground states. One which argues for a lower energy in periodically perturbed variants of the Laughlin wavefunction at $\nu=1 / 3$ is discussed in [12]. The presence of higher that expected peaks in numerical evaluations of the static structure factor was also treated in [13]. Another interesting result argued for the compatibility of the quantized Hall effect with the presence of crystalline structure in Quantum Hall crystals [14]. More recently [15], the possibility of an instability in the composite fermion description has been suggested based on calculations of the effective mass for these particles.

Special consideration requires the numerical evaluations for small systems [16]. It is clear that such calculations are essentially exact. It is also true that these evaluations 
predict a ground state separated from the first excited level by a gap in qualitatively agreement with the value predicted by the SMA. Therefore, we conclude that numerical results for small systems do not support any strong spontaneous breaking of translation symmetry in the ground state. However, numerical calculations do not seem to be able to rule out the presence of a weak charge density or correlation periodic structure which could affect the gap at various filling factors through natural Bragg reflection processes. The highest particle number considered up to now is near twelve. With such a small number of particles, weak periodic properties present in the ground state in the thermodynamic limit, could be hidden by boundary problems. It is possible that the calculated energy gap between the ground state and the first excited level could also be viewed as arising from a weakly periodic state in the thermodynamic limit.

From the experimental side, it is well known that finding traces of even the existence of the Wigner crystal phase with its strong charge density modulation is difficult. Therefore, the presence of a weak modulation in the properties of the ground state seems to be, at present, undetectable.

In the next section, the stability criterion is given, and in Section 3 its application to the SMA is considered.

\section{Stability Condition}

Let us consider the following hamiltonian describing 2D-electrons with coulomb interactions

$$
\begin{aligned}
\hat{H}= & \int d^{2} x \Psi^{+}(\vec{x}) \hat{O} \Psi(\vec{x})+\int d^{2} x\left(e \Psi^{+}(\vec{x}) \Psi(\vec{x})-\rho_{o}\right) \phi(\vec{x}) \\
& \int d^{2} x d^{2} y \Psi^{+}(\vec{y}) \Psi^{+}(\vec{x}) \frac{e^{2}}{\epsilon|\vec{x}-\vec{y}|} \Psi(\vec{x}) \Psi(\vec{y}) .
\end{aligned}
$$

The $\Psi$ fields satisfy the usual equal time commutation relations

$$
\left[\Psi(\vec{x}), \Psi^{+}(\vec{y})\right]_{+}=\delta^{(2)}(\vec{x}-\vec{y})
$$


which become in the first Landau level approximation of the QHE problem

$$
\left[\Psi(\vec{x}), \Psi^{+}(\vec{y})\right]_{+}=\Pi_{0}(\vec{x}-\vec{y}),
$$

for the specific fractional Hall effect system to be considered below . In (3), the $\Pi_{0}$ function is the projection operator onto the first Landau level. In this case, the free particle kinetic energy term determined by $\hat{O}$ in (1) will be absent as usual because the first Landau level restriction makes it a constant energy shift. A uniform background charge density $\rho_{o}$ is assumed as usual to assure the stability of the system without breaking translation invariance. A macroscopic dielectric constant $\epsilon$ is also considered for the medium in which the electronic system is imbedded.

The 2D-electron gas interacts with an electrostatic field $\phi(\vec{x})$ which is created by arbitrary but small fluctuations in the charge density of the background $\rho_{f}(\vec{x})$. The field $\phi(\vec{x})$ is therefore linked to $\rho_{f}(\vec{x})$ through

$$
\phi(\vec{x})=\int \frac{4 \pi}{\epsilon|\vec{x}-\vec{y}|} \rho_{f}(\vec{y}) d^{3} y .
$$

Assuming that the 2DEG is at a finite temperature determined by the parameter $\beta=(k T)^{-1}$, the total internal energy of the electronic system plus the fluctuating electrostatic field is given by

$$
U=<\hat{H}>+\int d^{3} x \frac{\epsilon}{8 \pi} \partial \phi(\vec{x}) \partial \phi(\vec{x}),
$$

where the statistical mean is considered in terms of the Bloch density matrix $\hat{\rho}$ and the partition function $Z$

$$
\begin{array}{r}
<\hat{O}>=\frac{\operatorname{Tr}(\hat{O} \hat{\rho})}{\operatorname{Tr}(\hat{\rho})}, \\
\hat{\rho}=\exp \left(-\frac{\hat{H}}{k T}\right), \\
Z=\operatorname{Tr}(\hat{\rho}) .
\end{array}
$$

After using the expression for the entropy [17]

$$
S=-<\ln \frac{\hat{\rho}}{Z}>=\frac{1}{k T}<\hat{H}>+k T \ln Z,
$$


the total Free-Energy of the electronic gas plus electrostatic fluctuations can be written in the following form

$$
\Gamma[T, \phi]=U-T S=\Gamma_{e}[T, \phi]+\int d x^{3} \frac{\epsilon}{8 \pi} \partial \phi(\vec{x}) \partial \phi(\vec{x})
$$

where the electronic contribution $\Gamma_{e}$ is given by

$$
\Gamma_{e}[T, \phi]=-\frac{1}{\beta} \ln [\operatorname{Tr}(\exp (-\hat{H} \beta))]
$$

The starting point of our analysis will be the condition that the work needed to be done at constant temperature to produce the arbitrary background charge density fluctuation $\rho_{f}(\vec{x})$ should always be positive for the system to be stable. Therefore, the second derivative of the Free-Energy functional over the field $\phi$ should be a positive definite kernel. Taking the second derivative of (10) gives the following relation

$$
\left.\frac{\delta^{2} \Gamma}{\delta \phi(\vec{x}) \delta \phi(\vec{y})}\right|_{\phi=0}=-\frac{\epsilon}{4 \pi} \partial^{2}(\vec{x}) \delta^{3}(\vec{x}-\vec{y})+\left.\frac{\delta^{2} \Gamma_{e}}{\delta \phi(\vec{x}) \delta \phi(\vec{y})}\right|_{\phi=0} \delta\left(x_{3}, y_{3}\right)
$$

where

$$
\delta\left(x_{3}, y_{3}\right)=\delta\left(x_{3}\right) \delta\left(y_{3}\right)
$$

The product of two $\delta$ functions in the coordinates orthogonal to the 2DEG plane appears because the functional derivatives over $\phi$ are taken in 3D-space while the electron gas is confined to the $x_{3}=0$ plane.

To evaluate the electronic contribution in (12), it is convenient to use the Matsubara approach by introducing temperature Heisenberg field variables

$$
\begin{aligned}
\Psi\left(\vec{x}, x_{4}\right) & =\exp \left(x_{4} \hat{H}\right) \Psi(\vec{x}) \exp \left(-x_{4} \hat{H}\right) \\
\Psi^{+}\left(\vec{x}, x_{4}\right) & =\exp \left(x_{4} \hat{H}\right) \Psi^{+}(\vec{x}) \exp \left(-x_{4} \hat{H}\right) .
\end{aligned}
$$

In terms of the particle density operator

$$
\rho\left(\vec{x}, x_{4}\right)=\Psi^{+}\left(\vec{x}, x_{4}\right) \Psi\left(\vec{x}, x_{4}\right)
$$


the second derivative of the electronic contribution to the Free-Energy at vanishing values of the field $\phi$ (where the mean value of the density is homogeneous) can be expressed in the following form

$$
\begin{aligned}
\frac{\delta^{2} \Gamma_{e}}{\delta \phi(\vec{x}) \delta \phi(\vec{y})}= & -\frac{1}{\beta} \int_{0}^{\beta} d x_{4} \int_{0}^{\beta} d y_{4}<T\left(e \rho\left(\vec{x}, x_{4}\right) \cdot e \rho\left(\vec{y}, y_{4}\right)-\rho_{o}^{2}\right)>\delta\left(x_{3}, y_{3}\right) \\
= & -\int_{0}^{\beta} d x_{4}<e \rho\left(\vec{x}, x_{4}\right) \cdot e \rho(\vec{y}, 0)-\rho_{o}^{2}>\delta\left(x_{3}, y_{3}\right) \\
= & -\int_{0}^{\beta} d x_{4}<e \exp \left(x_{4} \hat{H}\right) \rho(\vec{x}) \cdot e \exp \left(-x_{4} \hat{H}\right) \rho(\vec{x})>\delta\left(x_{3}, y_{3}\right) \\
& +\rho_{o}^{2} \beta \delta\left(x_{3}, y_{3}\right) .
\end{aligned}
$$

The energy eigenfunctions of the hamiltonian (1) satisfy

$$
\hat{H}\left|n>=\epsilon_{n}\right| n>
$$

and a completeness relation for them can be introduced after the density operator $\rho(\vec{x})$ in (17). This expression becomes

$$
\begin{aligned}
\frac{\delta^{2} \Gamma_{e}}{\delta \phi(\vec{x}) \delta \phi(\vec{y})}= & -\frac{e^{2} \delta\left(x_{3}, y_{3}\right)}{Z} \sum_{n, m \neq 0} \frac{\exp \left[\left(-\epsilon_{m} \beta\right)-\exp \left(-\epsilon_{n} \beta\right)\right.}{\epsilon_{n}-\epsilon_{m}}<n|\rho(\vec{x})| m><m|\rho(\vec{y})| n> \\
& -\frac{e^{2} \delta\left(x_{3}, y_{3}\right)}{Z} \beta<0|\rho(\vec{x})| 0><0|\rho(\vec{y})| 0>+\rho_{o}^{2} \beta \delta\left(x_{3}, y_{3}\right) .
\end{aligned}
$$

Let us consider now the zero temperature limit. As the temperature approaches zero the second term in (19) tends to cancel the constant contribution which is proportional to $\rho_{0}^{2}$ since the partition function $Z \rightarrow 1$ (a non degenerate ground state is assumed) and the mean value densities are constant being balanced by the background jellium. In the remaining sum, only terms having one of the indices $n$ or $m$ zero will survive if the ground state energy is nondegenerate. Therefore, (19) reduces to

$$
\left.\frac{\delta^{2} \Gamma_{e}}{\delta \phi(\vec{x}) \delta \phi(\vec{y})}\right|_{T \rightarrow 0}=-e^{2} \delta\left(x_{3}, y_{3}\right) \sum_{n \neq 0} \frac{2 R e[<0|\rho(\vec{x})| n><n|\rho(\vec{y})| 0>]}{\epsilon_{n}-\epsilon_{0}} .
$$

After substituting (20) in (12), the second derivative of the total Free-Energy in the zero temperature limit takes the form

$$
\begin{aligned}
\left.\frac{\delta^{2} \Gamma}{\delta \phi(\vec{x}) \delta \phi(\vec{y})}\right|_{T \rightarrow 0}= & -\frac{\epsilon}{4 \pi} \nabla_{\vec{x}}^{2} \delta^{(3)}(\vec{x}-\vec{y})- \\
& \delta\left(x_{3}, y_{3}\right) \sum_{n \neq 0} e^{2} \frac{2 R e[<0|\rho(\vec{x})| n><n|\rho(\vec{y})| 0>]}{\epsilon_{n}-\epsilon_{0}} .
\end{aligned}
$$


We restrict in what follows the fluctuation charge densities to those that are non vanishing only in the 2DEG plane $x_{3}=0$. In this case the associated electrostatic field $\phi$ can be expressed in the Fourier representation

$$
\phi(\vec{x})=\int \frac{d^{2} k}{(2 \pi)^{2}} \phi(\vec{k}) \exp \left(i \vec{k} \cdot \vec{x}-|\vec{k}|\left|x_{3}\right|\right)
$$

where $\phi(\vec{k})$ is any complex function of the momentum variables $\vec{k}$ in the plane satisfying $\phi(\vec{k})^{*}=\phi(-\vec{k})$.

The above property follows from acting with the 3D-laplacian on (22) which produces Poisson equation

$$
\nabla^{2} \phi(\vec{x})=-\int \frac{d^{2} k}{(2 \pi)^{2}} 2|\vec{k}| \phi(\vec{k}) \exp (i \vec{k} \cdot \vec{x}) \delta\left(x_{3}\right)=-4 \pi \rho_{f}(\vec{x})
$$

where $\rho_{f}(\vec{x})$ is the assumed arbitrary surface charge density fluctuation.

Using this class of potentials allows us to write the quadratic form

$$
\begin{aligned}
\Gamma^{(2)}[\phi] & =\int d^{3} x d^{3} y \phi(\vec{x}) \frac{\delta^{2} \Gamma}{\delta \phi(\vec{x}) \delta \phi(\vec{y})} \phi(\vec{y}) \\
& =\int \frac{d^{2} k}{(2 \pi)^{2}}\left(\frac{2|\vec{k}| \epsilon}{4 \pi}-K(\vec{k})\right) \phi^{*}(\vec{k}) \phi(\vec{k}),
\end{aligned}
$$

where the function $K$ is given by

$$
K(\vec{k})=\frac{2}{V} \sum_{n \neq 0} \frac{|<0| \rho_{\vec{k}}|n>|^{2}}{\epsilon_{n}-\epsilon_{o}}
$$

and $\rho_{\vec{k}}$ is the 2D-Fourier transform of the density operator.

Since the quadratic form (24) must be positive the following condition for the stability of the electronic gas arises

$$
1-\frac{2 \pi e^{2}}{\epsilon \hbar|\vec{k}|} \frac{2}{V} \sum_{n \neg 0} \frac{|<0| \rho_{\vec{k}}|n>|^{2}}{\epsilon_{n}-\epsilon_{o}} \geq 0 .
$$

This relation can be expressed in terms of the electron density $\rho=N / V$, the dynamical structure factor

$$
S(\vec{k}, w)=\frac{1}{N} \sum_{n \neq 0}|<0| \rho_{\vec{k}}|n>|^{2} \delta\left(w-\left(\epsilon_{n}-\epsilon_{o}\right) / \hbar\right), \quad(w>0)
$$


and the interaction potential

$$
v(\vec{k})=\frac{2 \pi e^{2}}{\epsilon \vec{k}}
$$

resulting in the final expression

$$
1-2 \rho v(\vec{k}) \int_{0}^{\infty} \frac{d w}{w} S(\vec{k}, w) \geq 0 .
$$

\section{Analysis of the SMA}

In this section, the condition (28) will used to considered the Girvin, MacDonald and Platzman (GMP) single mode approximation (SMA) for collective modes in the FQHE

ground states [5]. For this purpose, it is convenient to introduce the dimensionless wavevector

$$
\vec{q}=r_{o} \vec{k}
$$

and measure the energy in units of $\frac{e^{2}}{\epsilon r_{o}}$ where the magnetic radius is given in terms of the magnetic field intensity by $r_{o}=\sqrt{\frac{\hbar c}{|e B|}}$.

As discussed in [5], the SMA is characterized by a dynamic structure factor of the form

$$
S(\vec{q}, w)=\bar{s}(\vec{q}) \delta(w-\Delta(\vec{q}))
$$

where $\bar{s}$ is the static structure factor and $\Delta(\vec{q})$ is the collective mode energy as a function of the magneto-roton momentum.

In terms of the polarizability parameter

$$
\alpha(\vec{q})=\frac{\bar{s}(\vec{q})}{\Delta(\vec{q})},
$$

and the electron density filling factor $\nu$ :

$$
\rho=\frac{\nu}{2 \pi r_{o}^{2}},
$$

the stability condition for the approximate FQHE states described by the SMA can be written as

$$
\frac{|\vec{q}|}{2}-\nu \alpha(\vec{q}) \geq 0
$$


In order to investigate the validity of (31) for the SMA as applied to the main series of the $\nu=1 / m$ Laughlin states, we used the data for the polarizability parameter obtained in [5] for $\mathrm{m}=3,5,7,9$. The results indicate that the bound (31) is violated in a finite neighborhood of the maxima for the polarizability parameter as a function of the wavevector for all these states. The instability regions for $\nu=1 / 3$ and $\nu=1 / 5$ are shown in Figs. 1 and 2 as the zones of the curves lying above the straight lines. These lines are plots of the first term in (31) after multiplying by the factor $\mathrm{m}$ in each case. Similar results are valid for the $\nu=1 / 7$ and $\nu=1 / 9$ states. It can be seen that the instability is stronger for lower filling factors, as should be expected.

In conclusion, a criterion that the static dielectric response must satisfy for an interacting many body theory to be translational invariant was presented. From it, indications of an instability in the static response of the Laughlin states within the SMA approximation were obtained. These results suggest the existence of states different than the SMA variational states which could break continuous translation invariance and have energies lower than the Laughlin wavefunctions. Such wavefunctions could be similar to those discussed in [12 which we suspect are related to the special HartreeFock states discussed in [9], [10] and [11]. Alternatively, improvements of the original variational calculation of Girvin, MacDonald and Platzman may be possible. These problems will be considered in future work.

The relation obtained here may also be useful for other problems where the stability of an homogeneous many body system under spontaneous breaking of translation symmetry may be an issue.

\section{Acknowledgments.}

One of the authors (A.C.) greatly acknowledge the support of the High Energy Section of the ICTP for a helpful visit to the Centre and of the Condensed Matter Section for the nice opportunity to participate in the Adriatico Research Conference : "The 
Electron Quantum Liquid in Systems of Reduced Dimensions"(2-5 July 1996). The helpful remarks and comments of J. Strathdee, E. Tossati, S. Fantoni, G. Mussardo, J. Lawson, N. H. March, A. H. MacDonald, F. Ortolani, G. Fano and G. Baskaran are also greatly acknowledged.

We all are also deeply indebted to the Third World Academy of Sciences for its support through the TWAS Research Grant 93120 RG PHYS LA. 


\section{References}

[1] R.L. Willet, in: Physics of the Electron Solid, Ed. S.T. Chui (International Press, Boston 1994).

[2] N.H. March and M.P. Tosi, Coulomb Liquids, (Academic Press, 1984).

[3] L.D. Landau and E.M. Liftshitz, Electrodynamics of Continuos Media, (Pergamon Press, Oxford 1960).

[4] N.H. March and M.P. Tosi, Atomic Dynamics in Liquids, (MacMillan Ltd., 1976).

[5] S.M. Girvin, A.H. MacDonald and P.M. Platzman, Phys. Rev. B33, 2481 (1986).

[6] A. Perez Martinez, A. Cabo and V. Guerra, Preprint ICTP IC/95/295 (1995).

[7] A. Cabo and A. Perez Martinez, Preprint cond-mat/ 9601019 (1996).

[8] Q. Dai, J.L. Levy, A.L. Fetter, C.B. Hanna and R.B. Laughlin, Phys. Rev. B46 , $5642(1992-\mathrm{I})$.

[9] A. Cabo, Phys. Lett. A171, 90 (1992).

[10] A. Cabo, Phys. Lett. A191 , 323 (1994).

[11] A. Cabo, Phys. Lett. A211 , 297 (1996).

[12] S.T. Chui, T.M. Hakim and K.B. Ma, Phys. Rev. B33 , 7110 (1986).

[13] S.T. Chui, Phys. Rev. B32, 1436 (1985).

[14] Z. Tesanovic, F. Axel and B.I. Halperin, Phys. Rev. B39, 8525 (1989).

[15] S. Curnoe and P.C.E. Stamp, Preprint cond-mat/9601077 (1996).

[16] T. Chakraborty and P. Piatilainen, The Quantum Hall Effects ( Springer, Berlin 1994). 
[17] L.D. Landau, E.M. Lifshitz and L.P Pitaevski, Statistical Physics (Pergamon Press, Oxford 1980). 


\section{Figure Captions}

- Fig. 1 A plot of the SMA polarizability parameter as a function of the wavevector for $\nu=1 / 3$ Laughlin state. The momentum region in which the stability condition is not satisfied corresponds to the zone in which the curve is over the straight line.

- Fig. 2 The plot of the SMA polarizability parameter for the $\nu=1 / 5$ Laughlin state. The instability region is indicated by the wavevector neighborhood for which the curve passes over the straight line. 


\section{susceptibity parameter}

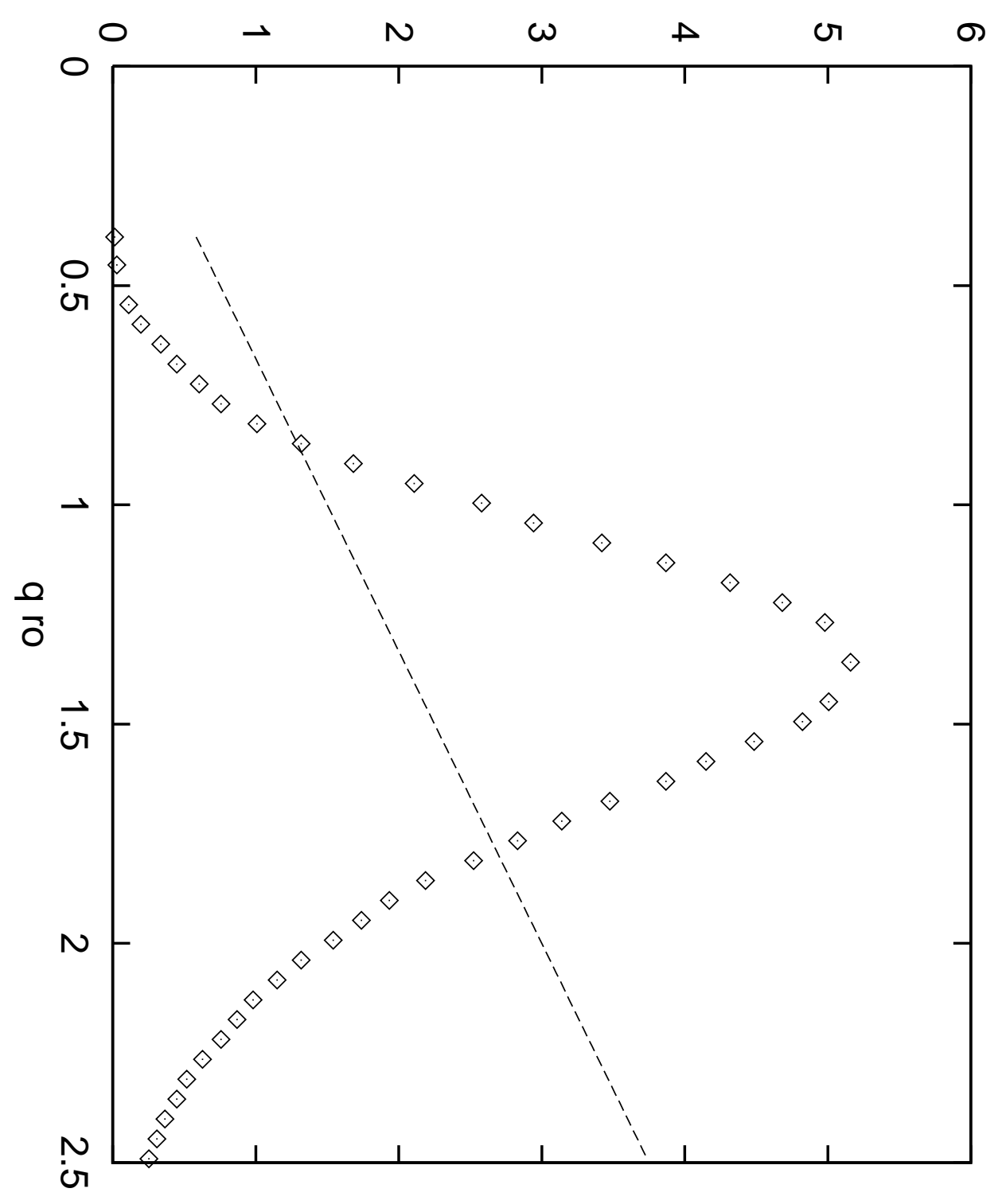




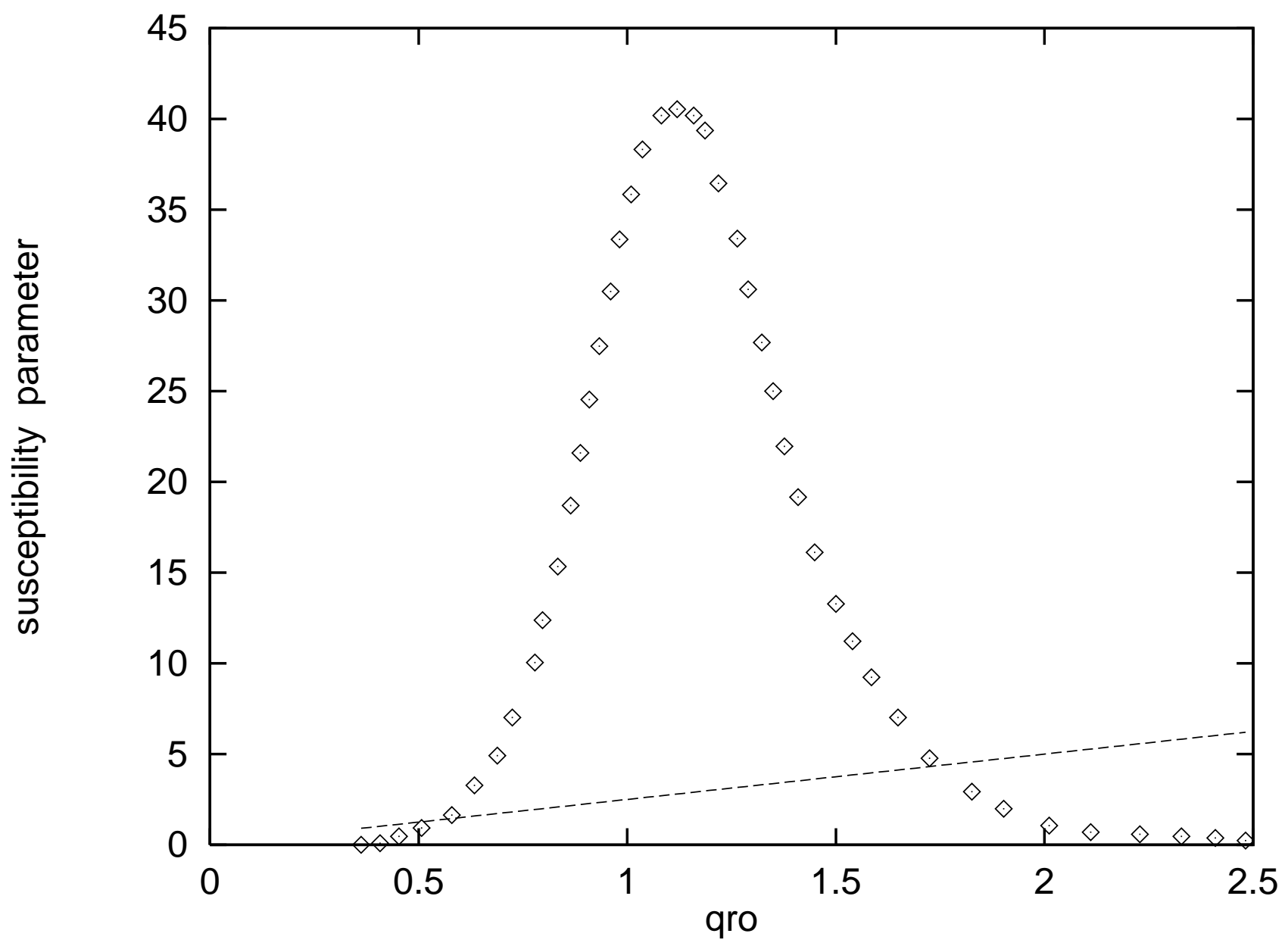

